\title{
Imagining and Preparing for the Aftermath of the COVID-19 Pandemic: A Justification for Taking Caring Responsibilities into Consideration when Allocating Scarce Resources
}

\author{
Christopher F.C. Jordens
}

Received: 30 April 2020 / Accepted: 27 August 2020

(C) Journal of Bioethical Inquiry Pty Ltd. 2020

\begin{abstract}
Various models have been used to "emplot" our collective experience of the COVID-19 pandemic, including the epidemiological curve, threshold models, and narrative. Drawing on a threshold model that was designed to frame resource-allocation decisions in clinical care, I offer an ethical justification for taking caring responsibilities into consideration in such decisions during pandemics. My basic argument is that we should prioritize the survival of patients with caring responsibilities for similar reasons we should prioritize the survival of healthcare professionals. More generally, the pandemic reveals the fundamental importance of informal care and affords an opportunity to raise questions of justice relating to it.
\end{abstract}

Keywords COVID-19 · Pandemic · Triage · Informal care $\cdot$ Justice $\cdot$ Narrative $\cdot$ Resource allocation

During the current COVID-19 pandemic, we must rely on the most effective control measures at our disposal: a combination of quarantine, self-isolation, social distancing, testing, and contact tracing. Behind these tried and tested practical measures lie the symbolic means of exerting control over epidemics, that is, the models we use to "emplot" (Mattingly 1994) our collective

C. F. Jordens $(\triangle)$

Sydney Health Ethics, Medical Foundation Building K25, The

University of Sydney, Sydney, NSW 2206, Australia

e-mail: chris.jordens@sydney.edu.au experience with a view to steering a course for it. I will consider three of these models in turn.

The first is a linear model that has exerted an enormous influence on public discourse and action during the pandemic: the epidemiological curve. The second is a threshold model that creates common ground for epidemiology and ethics, and which is exemplified by the ethical framework published elsewhere in this issue (Dawson et al. 2020). The third is narrative, which provides a resource for imagining and creating an aftermath to the pandemic.

I will use the second model to offer a justification for taking caring responsibilities into consideration when allocating scarce resources during pandemics. My basic argument is that we should prioritize the survival of patients with caring responsibilities for similar reasons we should prioritize the survival of healthcare professionals. More generally, the pandemic reveals the fundamental importance of informal care and affords an opportunity to raise questions of justice relating to it.

\section{The Epidemiological Curve}

The epidemiological curve emplots our collective experience as a peak we must ascend and, by implication, descend. It has been used by expert authorities to persuade publics to accept control measures in order to prevent healthcare systems from being overwhelmed, thereby precipitating the kind of scenarios I invoke below. The epidemiological curve presents an inherent problem when it comes to guiding purposive, future- 
directed collective action, however. It forces us to navigate an outbreak with our back to the future (so to speak) because the "flattening" of the curve becomes apparent only retrospectively. Confirmed cases register infections that occurred in the past - and how long in the past is a fact we only discover as the outbreak unfolds.

Furthermore, the model gives rise to a tension that is often framed, rightly or wrongly, as a trade-off between health outcomes and economic outcomes. If we abandon our control measures too early, we risk unleashing a second wave of infections (Xu and Li 2020). Those who accord primary value to human life typically regard this as a greater mistake than the alternative, which is to bear the costs of control measures for longer than (with the wisdom of hindsight) they might turn out to have been necessary.

\section{Threshold Models}

Threshold models take their bearings from "tipping points" at which the norms governing "business as usual" can with justification be supervened by a modified set of norms that come into play in exceptional circumstances. In the ethical framework published elsewhere in this issue (Dawson et al. 2020), tipping points occur wherever demand for an important resource outstrips supply. A similar model (Kain and Fowler 2019) expresses thresholds as a percentage of the normal capacity of healthcare systems. Apparently, it is normal to run these systems at 120 to 130 per cent capacity, but at 150 per cent we enter "code orange," which triggers the activation of pandemic preparedness measures. At 170 per cent of normal capacity, standards of care are modified and, beyond that, the logic of triage comes into play: patients who have a reasonable prospect of survival are turned away (the "walking wounded"), those who are expected to die get comfort care, and medical resources are focused on those who are most likely to benefit from them. As these resources become increasingly scarce, however, patients in the latter group are forced into a queue.

The ethical framework (Dawson et al. 2020) suggests that any existing moral consensus is at this point pulled in three different directions which can be broadly characterized as utilitarian, egalitarian, or geared to redressing discrimination and social disadvantage. Each of these approaches can be defended with reference to an established ethical literature on justice, but each yields responses that court objections from the others. For example, lottery-type solutions that can be justified by egalitarian principles are resisted by those who prioritize stewardship of resources. ${ }^{1}$ Similarly, measures that flow from egalitarian and utilitarian approaches court justifiable criticism on the grounds that the burdens of discrimination and disadvantage include the social determinants of ill-health; so if patients from disadvantaged groups are not "triaged out" on medical grounds, they enter queues or lotteries on an uneven playing field.

According to recent empirical research, public perceptions of fairness in Australia and New Zealand reflect trust in expert judgement and disfavour lottery-type solutions and considerations of social utility (Cheung et al. 2017). These findings obscure the degree to which expert judgements rest on utilitarian considerations that range from widely acceptable (e.g., number of lives saved) to contentious (e.g., number of life-years saved) to controversial. The latter include considerations not only of social utility but also of the quality of life saved, or "characteristics such as gender, ethnicity, sexuality, disability, religious or political views" (Dawson et al. 2020, Part B.5.a).

One consideration of social utility which appears not to attract much controversy is that of according priority to healthcare workers. Perhaps this is because it can be supported by several ethical justifications that have wide intuitive appeal. One justification appeals to a notion of fairness as reciprocity: it is a way to compensate for risks these workers assume in discharging their professional duty (Provincial COVID-19 Task Force 2020; National Ethics Advisory Committee 2007). Another justification is plainly utilitarian: this policy will enable healthcare workers to resume the struggle to save lives (Emanuel et al. 2020); in effect, they count as scarce resources too (Dawson et al. 2020). This justification holds so long as formal (professionalized, expert) systems supporting health and welfare remain intact. But what if such systems fail? This is an extreme scenario, but there have been intimations of this possibility in the aged care sector, in countries severely affected by the pandemic (BBC News 2020).

\footnotetext{
${ }^{1}$ This is a personal observation arising from my participation in drafting the ethics framework (Dawson et al. 2020) in consultation with intensive care clinicians, who are responsible for allocating resources for treating respiratory failure.
} 
One answer is that we must fall back onto informal care, by which I mean "care that is provided to the very young, the very old, the weak, the poor, and the sick by family, friends, neighbors, and concerned citizens, rather than by trained, licensed, or certified health care professionals" (Medical Dictionary 2020). This provides a utilitarian rationale for prioritizing those with caring responsibilities when allocating scarce resources during pandemics. Just as we have a collective interest in sustaining formal healthcare systems, we also have a collective interest in sustaining informal care.

This proposal is open to the objection that unlike care provided by healthcare professionals, informal care encompasses a broad and indefinite range of roles and activities and so would not be a useful consideration in prioritizing access to scarce resources. Whilst the meaning of "informal care" clearly needs to be scoped, this is not a reason to set this consideration aside altogether. Caring roles and activities that should be considered "within scope" include those that are not easily replaced or covered. For example, some lay carers acquire specialized skills to care for someone with particular needs, such as an elderly parent or partner, or someone with a disability. Some single parents with dependent children might not have an extended family or community that can take over their role if they fall sick and die. My point is that some consideration should be given to relationships of dependency that stand to be disrupted by the loss of informal care. By maximizing the survival chances of those with "lay" caring responsibilities as the pandemic unfolds, we also maximize the survival chances of those who depend on that care entirely should expert systems fail.

This measure does not discriminate by using personal or social characteristics as the basis for resource allocation decisions. To be a carer (either professional or lay) is not a characteristic; it is a social role. Insofar as the role of lay carer is associated with a personal characteristic, it is surely that of gender; and insofar as caring is associated with a social group that has experienced discrimination, it is surely women. If women happen to be advantaged by a consideration that prioritizes those with caring responsibilities, this can also be understood as going some way to redressing the social disadvantages women have experienced historically by carrying a greater responsibility for the work of caring for others. And not only historically: these disadvantages are amplified by the pandemic (Lewis 2020; Workplace Gender Equality Agency 2020).
One final point to make about threshold models is that they do not foreclose on the outcome of a pandemic. They are consistent with a good outcome, such as flattening the epidemic curve and descending the slope that lies beyond its peak. They are also consistent with a bad outcome like a failure of formal healthcare systems. They falter, however, in the face of the worst outcome of all, which would be a failure of both formal healthcare systems and informal care. This is the kind of scenario that survivalists prepare for, and it is theorized by sociologists as a state of anomie. We cannot specify a set of social norms for a state of exception which is characterized by a lack of social norms. For this we are forced to fall back on a different source of models to imagine our collective experience.

\section{Narratives}

Cultural narratives provide a rich resource for imagining the collapse of human civilizations. According to the scripts of apocalyptic and postapocalyptic fiction, lockdowns are interpreted as a stage of creeping authoritarianism that presages such a collapse. A recent spike in the sale of firearms in the United States illustrates how these scripts can exert a considerable influence on actual behaviour (Volpe 2020). Survivalist scripts also explain the behaviour of Australian consumers who began hoarding what was perceived to be essential supplies (including toilet paper) early in the pandemic and in the process showed how "prepping" can precipitate the very shortages for which it is adopted as a solution.

Narratives also provide a model for imagining the restoration of a social order in the aftermath of natural disasters and pandemics. According to the classic sociolinguistic formulation (Labov and Waletzky [1967] 1997), narrative structures are characterized by disruption of an existing social equilibrium (complicating action) and the restoration of that equilibrium (resolution). Some argue that a "return to normal" is not possible because the COVID-19 pandemic will permanently alter the conditions of social life. Others envisage a return to a "new normal." The degree to which this differs from the "old normal" invites us to consider how we might we use the pandemic as an opportunity to reimagine social norms and reshape society accordingly. As the epidemic curve flattens out in Australia, 
however, the narrative model is increasingly deployed to press a more mundane question: "When can we resume business as usual?"

\section{Questions of Justice}

Of the three main models we use to emplot our collective experience during the COVID-19 pandemic, only one - the threshold model - does not foreclose on the aftermath. I have used this model to fashion an argument that anticipates a threshold that Australia and New Zealand now seem unlikely to reach due to the successful mobilization of the first model - the epidemiological curve. Due to wide adoption of control measures, we have, in the main, preserved the integrity of our formal healthcare systems. Nevertheless, it is worth entertaining an alternative possibility we have perhaps only glimpsed: having no option other than to fall back on informal care. Informal care is also part of our social safety net that can and should be protected, including by the policies we adopt during the pandemic. The COVID-19 pandemic also offers an opportunity to raise questions of justice about informal care. Who has borne and continues to bear its burdens and its benefits (Jaggar 2001)?

\section{References}

BBC News 2020. Coronavirus: Spanish army finds care home residents "dead and abandoned." March 24. https://www. bbc.com/news/world-europe-52014023. Accessed April 29, 2020.

Cheung W., J. Myburgh, S. McGuinness, et al. 2017. A crosssectional survey of Australian and New Zealand public opinion on methods to triage intensive care patients in an influenza pandemic. Critical Care and Resuscitation 19(3): 254 265.

Dawson A., D. Isaacs, M. Jansen, et al. 2020. An ethics framework for making resource allocation decisions within clinical care: Responding to COVID-19. Journal of Bioethical Inquiry 17(4): https://doi.org/10.1007/s11673-020-10007-w.
Emanuel E.J., G. Persad, R. Upshur, et al. 2020. Fair allocation of scarce medical resources in the time of Covid-19. The New England Journal of Medicine 382(21): 2049-2055.

Jaggar, A. 2001. Feminism and the objects of justice. In Social and Political Philosophy: Contemporary Perspectives, edited by J.P. Sterba, 132-141. London: Routledge.

Kain, T., and R. Fowler. 2019. Preparing intensive care for the next pandemic influenza. Critical Care 23(1): 337.

Labov, W., and J. Waletzky. 1997 (1967). Narrative analysis: Oral versions of personal experience. Journal of Narrative and Life History 7(1-4): 3-38.

Lewis, H. 2020. The coronavirus is a disaster for feminism. The Atlantic, March 19. https://www.theatlantic. com/international/archive/2020/03/feminism-womensrights-coronavirus-covid19/608302/. Accessed April 29, 2020.

Mattingly, C. 1994. The concept of therapeutic "emplotment." Social Science \& Medicine 38(6): 811-822.

Medical Dictionary. 2020. s.v. “informal care.” https://medicaldictionary.thefreedictionary.com/informal+care. Accessed April 29, 2020.

National Ethics Advisory Committee. 2007. Getting through together: Ethical values for a pandemic. Ministry of Health, Wellington, New Zealand. https://neac.health.govt. $\mathrm{nz} /$ system/files/documents/publications/getting-throughtogether-jul07.pdf. Accessed June 2, 2020.

Provincial COVID-19 Task Force. 2020. COVID-19 ethics analysis: What is the ethical duty of health care workers to provide care during COVID-19 Pandemic? British Columbia Centre for Disease Control, British Columbia Ministry of Health. March 28. https://www2.gov.bc. ca/assets/gov/health/about-bc-s-health-care-system/officeof-the-provincial-health-officer/covid-19/duty_to_care during_covid_march_28_2020.pdf. Accessed April 29, 2020.

Volpe, A. 2020. A legacy of the COVID-19 pandemic will be that even more Americans own guns. Rolling Stone, April 8. https://www.rollingstone.com/culture/culture-features/covid19-coronavirus-pandemic-nra-gun-firearm-ownership980341/. Accessed April 29, 2020.

Workplace Gender Equality Agency. 2020. Gendered impacts of COVID-19. Australian Government, Sydney. https://www. wgea.gov.au/sites/default/files/documents/Gendered $\% 20$ impacts\%20of\%20COVID19_0.pdf. Accessed June 2, 2020.

$\mathrm{Xu}, \mathrm{S}$., and Y. Li. 2020. Beware the second wave of COVID-19. The Lancet 395(10233): 1321-1322.

Publisher's note Springer Nature remains neutral with regard to jurisdictional claims in published maps and institutional affiliations. 